SU-ITP-93-10

hepth/9305030

May 1993

\title{
Tachyon Hair on Two-Dimensional Black Holes ${ }^{\star}$
}

\author{
Amanda Peet ${ }^{\dagger}$, Leonard Susskind and Lárus Thorlacius ${ }^{\ddagger}$ \\ Department of Physics \\ Stanford University \\ Stanford, CA 94305
}

\begin{abstract}
Static black holes in two-dimensional string theory can carry tachyon hair. Configurations which are non-singular at the event horizon have non-vanishing asymptotic energy density. Such solutions can be smoothly extended through the event horizon and have non-vanishing energy flux emerging from the past singularity. Dynamical processes will not change the amount of tachyon hair on a black hole. In particular, there will be no tachyon hair on a black hole formed in gravitational collapse if the initial geometry is the linear dilaton vacuum. There also exist static solutions with finite total energy, which have singular event horizons. Simple dynamical arguments suggest that black holes formed in gravitational collapse will not have tachyon hair of this type.
\end{abstract}

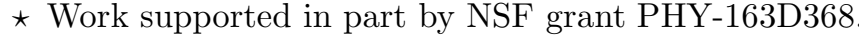

$\dagger$ e-mail: peet@dormouse.stanford.edu

$\ddagger$ e-mail: larus@dormouse.stanford.edu
} 
I.

The classical "no-hair theorems" of general relativity severely restrict the kinds of fields that can exist outside a black hole in four spacetime dimensions [1]. For example, in a static geometry with a smooth event horizon the only fields which are well behaved both asymptotically and on the event horizon are monopole gravitational and electromagnetic fields. Continuum string theory in two spacetime dimensions has classical solutions which are analogs of black holes [2,3] and provides a simplified context in which to study black hole physics. It has been suggested that the analog of the no-hair theorem for scalar fields does not hold for these two-dimensional geometries. In particular, it was pointed out by Witten [3] that the tachyon field of the two-dimensional string theory has a one-parameter family of solutions which are non-singular at the black hole event horizon. Subsequently, a number of authors have considered the back-reaction of the tachyon field on the black hole geometry using various approximation schemes [4-7]. However, no consensus has been reached as to whether the back-reaction destabilizes the configurations with tachyon hair and leads to singularities at the event horizon.

In this note we will examine the issue of tachyon hair by investigating solutions of the low-energy equations of motion of two-dimensional string theory with a non-vanishing tachyon background. We define a black hole with tachyon hair to be a geometry with a smooth event horizon and a tachyon field which is non-singular at that horizon. We find that solutions with hair exist, even when the tachyon back-reaction on the geometry is taken into account, but that they have non-vanishing asymptotic energy density and therefore infinite ADM mass. These static geometries with hair correspond to eternal black holes where there is a balance between an outgoing and an incoming energy flux. The outgoing flux emerges from the past singularity and will not be present when a black hole is formed in gravitational collapse. We therefore conclude that dynamically formed black holes cannot have tachyon hair of this type. When the tachyon back-reaction is included, there also exist solutions with diverging string coupling and a curvature singularity at the horizon [7]. We argue that such configurations will not arise in dynamical evolution from non-singular initial data.

The issue of black hole hair comes up in attempts to describe black hole geometries in the context of the $c=1$ matrix model [8]. It is important to resolve whether one should look for a family of black holes labelled by other parameters besides the mass. At the end we will comment on the possible relevance of our results to this issue.

II.

Tree-level backgrounds in string theory are determined by the conditions of Weyl invariance of the worldsheet sigma model. These can be obtained as the variational equations of an effective action for the target space metric, dilaton and tachyon fields [9], which has

$\S$ One can take the view that this type of tachyon hair defines superselection sectors in the theory since the amount of hair cannot be changed in dynamical processes. In each sector the (infinite) energy associated with the asymptotic tachyon field could then be subtracted off to obtain a finite mass for a given configuration. 
the following form in two dimensions $"$,

$$
S=\frac{1}{2 \pi} \int d^{2} x \sqrt{-g} e^{-2 \phi}\left\{R+4(\nabla \phi)^{2}+8-\frac{1}{2}(\nabla T)^{2}-V(T)+\cdots\right\},
$$

The tachyon potential $V(T)=-T^{2}+c T^{3}+\ldots$ is non-linear and only the leading term, which determines the tachyon mass, is universal. The higher order terms depend on the renormalization prescription used to define the worldsheet theory $[10,11]$ but our conclusions will not be affected by their detailed form. The effective action receives corrections from worldsheet perturbation theory, which are non-linear in the sigma model fields and of higher order in derivatives. We expect our qualitative conclusions to hold when those corrections are included. There will also be corrections from string loops which contribute terms of higher order in the string coupling $e^{\phi}$. These will lead to interesting and important quantum effects such as Hawking radiation. In this work we will only consider string theory at tree level. The question of whether or not classical two-dimensional black holes can have tachyon hair is relevant to the evolution of massive black holes in the quantum theory. One would, for example, expect the presence of black hole hair to affect the Hawking temperature.

The classical equations of motion obtained from the action (1) can be written,

$$
\begin{aligned}
\nabla^{2} T-2 \nabla \phi \cdot \nabla T & =V^{\prime}(T), \\
\nabla^{2} \phi-2(\nabla \phi)^{2} & =-4+\frac{1}{2} V(T), \\
2 \nabla_{\mu} \nabla_{\nu} \phi-g_{\mu \nu} \nabla^{2} \phi & =\frac{1}{2} \nabla_{\mu} T \nabla_{\nu} T-\frac{1}{4} g_{\mu \nu}(\nabla T)^{2} .
\end{aligned}
$$

Note that the tachyon source terms in the equations for the metric and dilaton are at least quadratic in $T$ while the tachyon equation itself is linear in $T$ to leading order. This means that one can consistently view a weak tachyon field as a small perturbation and ignore its back-reaction on the background geometry to leading order. We will come back to the issue of the back-reaction due to a finite strength tachyon field later on. Let us begin by looking for the static solutions of the dilaton-gravity sector in the absence of tachyon matter. They are conveniently described using the two-dimensional analog of Schwarzschild coordinates, for which the metric has the form,

$$
d s^{2}=-f(r) d t^{2}+\frac{1}{f(r)} d r^{2} .
$$

The equations in vacuum for $f$ and $\phi$ reduce to,

$$
\phi^{\prime \prime}=0 \quad, \quad f^{\prime} \phi^{\prime}-2 f \phi^{2}+4=0 .
$$

The dilaton is a linear function of the spatial coordinate and any constant part can be absorbed into a shift of $\phi$. After rescaling the coordinates to make the metric approach the

9 We take $\alpha^{\prime}=2$ throughout. 
flat Minkowski metric asymptotically we are left with a one-parameter family of physically distinct static geometries,

$$
\phi(r)=-\sqrt{2} r \quad, \quad f(r)=1-\mu e^{-2 \sqrt{2} r} .
$$

All these solutions approach the linear dilaton vacuum in the asymptotic region $r \rightarrow$ $+\infty$. The parameter $\mu$ is proportional to the mass of the black hole as determined by the canonical ADM procedure, $\mu=\sqrt{2} \pi M_{A D M}$. For positive mass the geometry has a spacelike curvature singularity inside an event horizon at $r_{h}=\frac{1}{2 \sqrt{2}} \log \left(\frac{1}{\mu}\right)$ but for negative mass the singularity is naked.

We now wish to consider a weak static tachyon field outside a positive mass black hole. For this purpose it is convenient to absorb a factor of the string coupling into the tachyon field,

$$
U=e^{-\phi} T
$$

Then the tachyon equation of motion becomes

$$
\nabla^{2} U+\left(\nabla^{2} \phi-(\nabla \phi)^{2}\right) U=e^{-\phi} V^{\prime}\left(e^{\phi} U\right)
$$

The redefined tachyon field has a standard kinetic term and it is $U$ which is the physical propagating mode in the system. The mass term for $U$ depends on the background geometry. It reduces to zero in the linear dilaton vacuum, as expected, and its effect therefore vanishes in the asymptotic region.

The linearized equation for a static $U$ field on a black hole background in our Schwarzschild coordinates is

$$
\left(1-\mu e^{-2 \sqrt{2} r}\right) U^{\prime \prime}+2 \sqrt{2} \mu e^{-2 \sqrt{2} r} U^{\prime}-2 \mu e^{-2 \sqrt{2} r} U=0 .
$$

It is straightforward to find the general solution for $U$ outside the black hole event horizon [12]. The change of variables $y=\mu e^{-2 \sqrt{2} r}$ takes (8) to a hypergeometric equation,

$$
y(1-y) \frac{d^{2} U}{d y^{2}}+(1-2 y) \frac{d U}{d y}-\frac{1}{4} U=0,
$$

with regular singular points at $y=0$ and $y=1$. The asymptotic region of the black hole geometry is obtained as $y \rightarrow 0^{+}$and the event horizon is approached as $y \rightarrow 1^{-}$. The general solution of (9) is

$$
U(y)=A_{1} u_{1}(y)+A_{2} u_{2}(y)
$$

where the basic solutions are given in terms of a single hypergeometric function,

$$
u_{1}(y)=F\left(\frac{1}{2}, \frac{1}{2} ; 1 ; y\right) \quad, \quad u_{2}(y)=F\left(\frac{1}{2}, \frac{1}{2} ; 1 ; 1-y\right) \text {. }
$$

The hypergeometric function is analytic in the interval $0<y<1$, which corresponds to 
the black hole exterior, and has the following asymptotic behavior near the endpoints,

$$
\begin{aligned}
F\left(\frac{1}{2}, \frac{1}{2} ; 1 ; y\right) & \sim 1+\frac{1}{4} y+O\left(y^{2}\right) \quad \text { as } y \rightarrow 0, \\
F\left(\frac{1}{2}, \frac{1}{2} ; 1 ; 1-y\right) & \sim-\frac{1}{\pi} \log \left(\frac{y}{4}\right)\left(1+\frac{1}{4} y+O\left(y^{2}\right)\right)-\frac{1}{2 \pi} y+O\left(y^{2}\right) \text { as } \quad y \rightarrow 0,
\end{aligned}
$$

Any solution with a non-vanishing coefficient of $u_{1}$ in (10) has a logarithmic singularity at the event horizon. Tachyon hair must therefore be a pure $u_{2}$ configuration which is smooth at $y=1$ but behaves logarithmically for small $y$. Going back to the Schwarzschild coordinates we see that the hair is linear far away from the black hole,

$$
U(r) \sim \beta r \quad \text { as } \quad r \rightarrow \infty
$$

Since the mass of $U$ goes to zero in the asymptotic region it is reasonable that static solutions approach linear behavior there. Because of the non-vanishing gradient of $U$ there is a finite energy density in the asymptotic region, $\mathcal{E}=\frac{\beta^{2}}{2 \pi}$, which means that the total ADM mass of a two-dimensional black hole with tachyon hair is divergent. One might expect this infinite energy to lead to a disastrous back-reaction on the geometry but this does not happen because the coupling strength $e^{\phi}$ goes to zero asymptotically, due to the linear dilaton, and the matter decouples from the gravitational sector. Because we are working in two spacetime dimensions such solutions can be given an interpretation in terms of balancing incoming and outgoing energy fluxes carried by (zero-momentum) tachyons. To see this, write the linear $U$ field in terms of null coordinates, $U\left(r^{ \pm}\right)=\frac{\beta}{2}\left(r^{+}-r^{-}\right)$, and observe that $T_{++}=T_{--}=\frac{\beta^{2}}{4 \pi}$. Usually one associates a non-vanishing energy flux with a propagating field, and one can obtain the above linear $U$ field as the infinite wavelength limit of such an oscillating mode.

A solution built out of $u_{2}$ alone is perfectly smooth at the event horizon and can be extended through it into the black hole interior. This can be seen by making a coordinate transformation to the two-dimensional analog of Kruskal-Szekeres coordinates, $U, V$, in which the black hole geometry is manifestly regular at the event horizon,

$$
d s^{2}=\frac{d U d V}{2 U V-\mu} .
$$

A static geometry will only depend on the product $\xi=-2 U V$. The relationship between $\xi$ and our spatial variable $y$ is

$$
y=\frac{1}{1+\frac{\xi}{\mu}},
$$

and it is non-singular at the event horizon. This means that a solution which is regular as $y \rightarrow 1$ will also be regular as a function of $\xi \sim 0$ and can be extended into the black hole interior to negative values of $\xi$. This indicates that a flux interpretation of the solutions with tachyon hair involves fluxes which pass through the event horizon and terminate 
(originate) on the future (past) singularity. If, on the other hand, the black hole is formed dynamically by gravitational collapse there is no past singularity in the geometry and the tachyon hair would have to be supported by an outgoing flux emerging from the original vacuum. ${ }^{\star}$ This would require imposing non-standard boundary conditions on the system at an early time, before the collapse takes place, and we conclude that dynamically formed black holes cannot carry tachyon hair of this type.

The ability of eternal black holes in two-dimensional string theory to carry tachyon hair relies on the particular manner in which the tachyon field is coupled to the gravitational theory. Consider for comparison the model suggested by Callan et al. [13] for the study of black hole physics in two dimensions. The classical gravity sector in this theory is identical to the leading terms in the two-dimensional string theory (1) so in the absence of matter it has the same classical black hole solutions (5). The matter sector consists of a set of conformally coupled scalar fields which replace the tachyon of the string theory. In Schwarzschild coordinates the classical equation of motion of a conformal scalar field, $a$, is

$$
f a^{\prime \prime}+f^{\prime} a^{\prime}=0
$$

and the general solution in a black hole background is given by

$$
a(r)=a_{0}+a_{1} \log \left(e^{2 \sqrt{2} r}-\mu\right) .
$$

The only solution which is regular at the event horizon is a constant which means that black holes cannot have non-trivial scalar hair in this two-dimensional model.

III.

Will our conclusions be qualitatively changed by higher order corrections to the effective action (1)? Let us first consider contributions from higher orders in sigma model perturbation theory. The static black hole solution (5) is only valid to lowest order but it has been suggested that an exact solution to the Weyl invariance conditions on the metric and dilaton, in the absence of a tachyon background, is provided by a deformed "cigar" geometry [14], for which the detailed spatial dependence of $\phi$ and $f$ differs from (5) but there is still a smooth horizon and the fields approach the linear dilaton vacuum asymptotically.

It is easy to see that near a smooth event horizon the linearized scalar field equation always has one non-singular solution and one which diverges logarithmically. Asymptotically far away the mass term goes to zero and the general solution is a linear function of the Schwarzschild coordinate. For the case of a lowest order black hole background (5) we were able to interpolate between the event horizon and asymptotic region with the exact solution (10) and we found that solutions which are smooth at the event horizon are in fact orthogonal to those that are well behaved asymptotically. While this exact orthogonality will presumably not survive to higher orders it is clear that the corrections

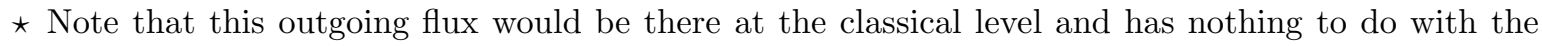
quantum mechanical Hawking flux which emanates from a black hole. 
will not generically change the fact that solutions, which are smooth at the event horizon, will have a non-vanishing energy density at infinity.

What about the back-reaction of the tachyon field on the geometry? S. K. Rama has argued that the effect of the back-reaction is to destabilize any smooth solution at the horizon [7]. This would of course rule out the existence of any tachyon hair in the full theory, even if one is willing to work with solutions which have non-vanishing asymptotic energy density. However, the analysis of reference [7] is incomplete. While one can indeed find static solutions of (2) with non-vanishing $T$, and a curvature singularity along with diverging string coupling at the event horizon, there also exist configurations with nonvanishing tachyon hair which have a perfectly smooth event horizon. Let us again work in a Schwarzschild coordinate system (3) and consider the full set of static equations,

$$
\begin{aligned}
f T^{\prime \prime}+f^{\prime} T^{\prime}-2 f \phi^{\prime} T^{\prime} & =V^{\prime}(T), \\
f \phi^{\prime \prime}+f^{\prime} \phi^{\prime}-2 f \phi^{\prime 2} & =-4+\frac{1}{2} V(T), \\
\phi^{\prime \prime} & =\frac{1}{4} T^{2} .
\end{aligned}
$$

Let $x=r-r_{h}$ be the coordinate distance from the event horizon and look for a regular solution of the form,

$$
\begin{aligned}
T(x) & =\sum_{k=0}^{\infty} t_{k} x^{k}, \\
\phi(x) & =\sum_{k=0}^{\infty} \phi_{k} x^{k}, \\
f(x) & =\sum_{k=1}^{\infty} f_{k} x^{k},
\end{aligned}
$$

which is expected to be valid near the event horizon. The power series coefficients can be determined by inserting (19) into (18) and working order by order in $x$. We choose to parametrize the family of solutions by the tachyon and dilaton fields at the event horizon and we use the freedom to rescale the coordinate $x$ to fix the ratio between $f_{1}$ and $f_{2}$ as -1 . The leading terms in the solution are then given by,

$$
\begin{aligned}
& T(x)=t_{0}+\frac{V^{\prime}\left(t_{0}\right)}{4-\frac{1}{2} V\left(t_{0}\right)} x+\frac{\frac{1}{4} V^{\prime}\left(t_{0}\right) V^{\prime \prime}\left(t_{0}\right)}{\left(4-\frac{1}{2} V\left(t_{0}\right)\right)^{2}} x^{2}+\ldots, \\
& \phi(x)=\phi_{0}-x+\frac{\frac{1}{8} V^{\prime}\left(t_{0}\right)^{2}}{\left(4-\frac{1}{2} V\left(t_{0}\right)\right)^{2}} x^{2}+\ldots, \\
& f(x)=\left(4-\frac{1}{2} V\left(t_{0}\right)\right)\left(x-x^{2}\right)+\ldots
\end{aligned}
$$

When $T=0$ this gives us the leading terms in the expansion of the black hole solution (5) (up to a rescaling of the coordinate by $\sqrt{2}$ ). The solution will behave in some complicated 
way as we move away from the horizon but the asymptotic region is governed by the linear dilaton and the solution will match onto a linear $U$ field there. Thus there exist infinite energy configurations with tachyon hair which have a non-singular event horizon.

There are other solutions, where the tachyon back-reaction leads to a singular event horizon. The dominant small $x$ behavior can be parametrized as follows,

$$
\begin{aligned}
& T(x)=\eta \log x, \\
& \phi(x)=\phi_{0}-\frac{\eta^{2}}{4} \log x, \\
& f(x)=x^{1-\frac{\eta^{2}}{2}} .
\end{aligned}
$$

The scalar curvature blows up as the horizon is approached, $R \sim \frac{\eta^{2}}{2}\left(1-\frac{\eta^{2}}{2}\right) x^{-1-\frac{\eta^{2}}{2}}$, and the string coupling also diverges there, $e^{\phi} \sim e^{\phi_{0}} x^{-\frac{\eta^{2}}{2}}$.

These singular solutions presumably correspond to the ones discussed in reference [7]. Can they be the end product when a two-dimensional black hole is formed in gravitational collapse? Consider a set of initial data describing infalling tachyon matter and assume that after some time a black hole forms with a spacelike curvature singularity inside an apparent horizon. A singular horizon might develop from an instability in the evolution of the fields at or near the apparent horizon, which would lead to a new curvature singularity separate from the one in the black hole interior. This scenario can be ruled out using the classical equations of motion. For this we adapt a conformal gauge argument previously given [17] in the context of the dilaton gravity model of Callan et al. [13]. Let $\sigma^{ \pm}$be a set of null conformal coordinates; $d s^{2}=-e^{2 \rho(\sigma)} d \sigma^{+} d \sigma^{-}$. In this gauge the second equation in (2) becomes

$$
\partial_{+} \partial_{-} \phi-2 \partial_{+} \phi \partial_{-} \phi=e^{2 \rho}\left(1-\frac{1}{8} V(T)\right) .
$$

The incoming matter is initially described by some left-moving tachyon field in the asymptotic weak-coupling region and we'll assume that there is a null line, $\sigma^{+}=\sigma_{0}^{+}$, below which we have the vacuum configuration (see figure 1a). On this null line the dilaton field is a monotonic function of $\sigma^{-}$, increasing as we go deeper into the strong coupling region. The dilaton diverges at the event horizon in the singular static solution (21). In order for such a blow-up to occur, away from the strong-coupling singularity in the black hole interior, the dilaton must first develop a local maximum, which then has to grow without bound. But if the dilaton field is a monotonic function of $\sigma^{-}$on some null line of constant $\sigma^{+}=\sigma_{0}^{+}$then the equation of motion (22) ensures that it will remain monotonic on all null lines $\sigma^{+}>\sigma_{0}^{+}$. If a local maximum were to occur it would have to be accompanied by a local minimum closer to the strong coupling region, as illustrated in figure $1 \mathrm{~b}$. Call the points where the extrema occur $\sigma_{1}^{-}\left(\sigma^{+}\right)$and $\sigma_{2}^{-}\left(\sigma^{+}\right)$. At these points $\partial_{-} \phi$ vanishes and (22) reduces to

$$
\partial_{+}\left(\partial_{-} \phi\right)=e^{2 \rho}\left(1-\frac{1}{8} V(T)\right)
$$

As long as the right hand side of (23) remains positive the two curves $\sigma_{1}^{-}\left(\sigma^{+}\right)$and $\sigma_{2}^{-}\left(\sigma^{+}\right)$ 
must approach each other as $\sigma^{+}$increases. The dilaton field is monotonic on $\sigma^{+}=\sigma_{0}^{+}$ so there would have to be a smallest value of $\sigma^{+}$where the extrema first occur and at which $\sigma_{1}^{-}=\sigma_{2}^{-}$. But, since the equations of motion prevent $\sigma_{1}^{-}$and $\sigma_{2}^{-}$from separating it follows that the dilaton must remain monotonic for all $\sigma^{+}>\sigma_{0}^{+}$. This conclusion is in agreement with the results of Russo [5] who studied black hole formation due to incoming tachyon matter and found no evidence for singular horizons.

Our argument would fail if the shape of the tachyon potential were such that $V(T)>8$ were possible for some value of $T$. This, however, is very unlikely since in order to become positive the tachyon potential would have to have a minimum somewhere and that would correspond to a stable vacuum of the bosonic string.

IV.

We have found that non-singular tachyon hair is a feature of eternal static black holes only and does not arise when the black hole is dynamically formed. We have also seen that new singularities, associated with non-vanishing tachyon hair of the type (21), cannot be formed at the horizon. We have not given proof that the original black hole singularity cannot merge with the apparent horizon to form a singular event horizon, which might carry singular tachyon hair. However, the nature of the curvature singularity at the event horizon in (21) appears to be quite different from that of the singularity inside a classical black hole (5), where the curvature blows up at the same rate as the coupling strength, $R=8 \mu e^{2 \phi}$. We find it more likely that the tachyon field will be absorbed by the black hole or radiated away as the geometry settles down to its final state.

The asymptotic linear $U$ field of a two-dimensional black hole with tachyon hair can be given an interpretation in terms of the fermion fields of the $c=1$ matrix model. The standard bosonization formulas identify $\partial_{r} U$ with a shift in the fermion number density. Non-singular tachyon hair of the type discussed above then corresponds to shifting the fermi level in the inverted harmonic oscillator potential of the matrix model. On the other hand, recent efforts to understand black holes in the context of the matrix model suggest that the height of the fermi level corresponds to the black hole mass $[12,15,16]$. These two interpretations appear to be in conflict, unless the mass and tachyon hair of static black holes in string theory are correlated and there is only a one parameter family of physically distinct solutions.

In conclusion we would like to emphasize once again that two-dimensional tachyon hair is very different from the hair envisioned in four-dimensional no-hair conjectures. Usually black hole hair refers to fields which might result from the collapse of matter to form the black hole whereas we find that tachyon hair on two-dimensional black holes is infinitely long and can neither be grown nor cut.

Note added: While preparing this manuscript we became aware of the work of N. Marcus and Y. Oz [18] which has some overlap with the present work.

* The tachyon potential has been computed to all orders in $T$ and found to be negative definite [11]. The form of the potential is of course highly sensitive to the sigma model renormalization prescription used, but we expect the feature that the potential does not turn around and become positive to be universal. 


\section{REFERENCES}

1. V. Ginzburg and L. Ozernoi, Soviet Phys., JETP 20 (1965), 689;

A. Doroshkevich, Ya. Zeldovich and I. Novikov, Soviet Phys., JETP 22 (1966), 122 ;

W. Israel, Phys. Rev. 164 (1967), 1776;, Comm. Math. Phys. 8 (1968), 245;

B. Carter, Phys. Rev. Lett. 26 (1971), 331;

J. D. Bekenstein, Phys. Rev. D5 (1972), 1239;

J. B. Hartle, in Magic without Magic, ed. J. Klauder (Freeman, 1972);

C. Teitelboim, Phys. Rev. D5 (1972), 2941;

R. H. Price, Phys. Rev. D5 (1972), 2419: ibid. 2439.

2. M. Mueller, Nucl. Phys. B337 (1990), 37;

S. Elitzur, A. Forge and E. Rabinovici, Nucl. Phys. B359 (1991), 581;

M. Rocek, K. Schoutens and A. Sevrin, Phys. Lett. B265 (1991), 303;

G. Mandal, A. Sengupta and S. R. Wadia, Mod. Phys. Lett. A6 (1991), 1685.

3. E. Witten, Phys. Rev. D44 (1991), 314.

4. S. P. de Alwis and J. Lykken, Phys. Lett. B269 (1991), 264.

5. J. G. Russo, Classical Solutions in Two-Dimensional String Theory and Gravitational Collapse, University of Texas preprint, UTTG-31-92, December 1992.

6. S. Chaudhuri and D. Minic, On the Black Hole Background of Two-Dimensional String Theory, University of Texas preprint, UTTG-30-92, December 1992.

7. S. K. Rama, Tachyon Back Reaction on $d=2$ Black Hole, Trinity College Dublin preprint, TCD-3-93, March 1993.

8. For a review of matrix models see P. Ginsparg and G. Moore, Lectures on 2D Gravity and 2D String Theory, preprint YCTP-P23-92, LA-UR-92- 3479.

9. C. G. Callan, D. Friedan, E. J. Martinec and M. J. Perry, Nucl. Phys. B262 (1985), 593

E. S. Fradkin and A. A. Tseytlin, Nucl. Phys. B271 (1986), 561;

C. G. Callan and Z. Gan, Nucl. Phys. B272 (1986), 647.

10. T. Banks, Nucl. Phys. B361 (1991), 166;

A. A. Tseytlin, Phys. Lett. B264 (1991), 311.

11. A. Cooper, L. Susskind and L. Thorlacius, Nucl. Phys. B363 (1991), 132; Quantum Cosmology on the Worldsheet, in Proceedings of the "Strings and Symmetries" workshop, Stony Brook, N. Y., May 20-25, 1991, W. Siegel et al. eds., (World Scientific).

12. S. R. Das, Mod. Phys. Lett. A8 (1993), 69.

13. C. G. Callan, S. B. Giddings, J. A. Harvey and A. Strominger, Phys. Rev. D45 (1992), R1005.

14. R. Dijkgraaf, E. Verlinde and H. Verlinde, Nucl. Phys. B371 (1992), 269.

15. G. Mandal, A. Sengupta and S. R. Wadia, Mod. Phys. Lett. A7 (1992), 3703. 
16. J. G. Russo, Phys. Lett. B300 (1993), 336.

17. L. Susskind and L. Thorlacius, Nucl. Phys. B382 (1992), 123.

18. N. Marcus and Y. Oz, The Spectrum of the 2D Black Hole, or Does the 2D black hole have tachyonic or W-hair?, Tel-Aviv preprint, TAUP-2046-93, May 1993. 